\title{
A NOTE ON NEARLY PARACOMPACTNESS
}

\author{
Ilija Kovačević*
}

\begin{abstract}
The purpose of the present paper is to study some properties of nearly paracompactness, $\alpha$-Hausdorff subset, almost closed mappings and closed graphs.
\end{abstract}

\section{Preliminaries}

Our notation is standard. No separation properties are assumed for spaces unless explicitly stated.

A subset $A$ of a space $X$ is regular open iff $\operatorname{Int} C l A=A$. A subset $A$ of a space $X$ is regular closed iff $C l \operatorname{Int} A=A,[13]$.

A set $P$ is said to be $\delta$-closed if for each point $x \notin P$, there exists an open set $G$ containing $x$ such that $\alpha(G) \cap P=\emptyset\left(\alpha(G)=\bar{G}^{0}\right)$. A set $G$ is $\delta$-open iff its complement is $\delta$-closed, [15].

For every topological space $(X, \tau)$, the collection of all $\delta$-open sets forms a topology for $X$, which is weaker than $\tau$. This topology has a base consisting of all regular open subsets in $(X, \tau)$. We shall denote this topology by $\tau^{*}$. An open cover $\mathcal{U}$ is even if there exists a neighborhood $V$ of the diagonal in $X \times X$ such that $V[x] \subset U,(V[x]=\{y:(x, y) \in V\})$ for some $U \in \mathcal{U}[2]$.

A subset $A$ of a space $X$ is $\alpha$-paracompact ( $\alpha$-nearly paracompact) with respect to a subset $B$ iff for every open (regular open) cover $\mathcal{U}=\left\{U_{i}: i \in I\right\}$ of $A$ there is an open family $\mathcal{V}=\left\{V_{j}: j \in J\right\}$ such that:

1. $\mathcal{V}$ refines $\mathcal{U}$

2. $A \subset \cup\left\{V_{j}: j \in J\right\}$,

3. $\mathcal{V}$ is locally finite at each point $x \in B$.

Subsets $A$ and $B$ of a space $X$ are mutually $\alpha$-paracompact ( $\alpha$-nearly paracompact) iff the subset $A$ is $\alpha$-paracompact ( $\alpha$-nearly paracompact) with respect to the subset $B$ and $B$ is $\alpha$-paracompact ( $\alpha$-nearly paracompact) with respect to the subset $A[8]$.

AMS (MOS) Subject Classification 1991. Primary: 54D10, 54D18.

Key words and phrases: compact, $\alpha$-Hausdorff, $\alpha$-nearly paracompact, $\alpha$ paracompact, $\alpha$-regular, almost closed mappings, closed graph, equivalence relation. 
A subset $A$ of a space $X$ is $\alpha$-Hausdorff iff for any two points $a, b$ of a space $X$, where $a \in A$ and $b \in X \backslash A$, there are disjoint open sets $U$ and $V$ containing $a$ and $b$ respectively.

A subset $A$ of a space $X$ is $\alpha$-regular ( $\alpha$-almost regular) iff for any point $a \in A$ and any open (regular open) set $U$ containing $a$, there is an open set $V$ such that $a \in V \subset C l V \subset U,[11]$.

A mapping $f: X \rightarrow Y$ is almost closed iff for every regular closed set $F$ in $X$, the set $f(F)$ is closed, [13].

A mapping $f: X \rightarrow Y$ has a closed graph $G(f)$ iff $G(f)=\{(x, f(x))$ : $x \in X\}$ is closed in $X \times Y,[1]$.

Let $X$ be any topological space. Let $(X, \rho)$ be a graph, where $\rho$ is an equivalence relation in $X$. Let $\rho(X)$ denote $\rho$-class of $x$. Let $X / \rho$ denote the set of all $\rho$-classes of equivalence relation $\rho$ in $X$.

A subset $A$ of a space $X$ is admissible if it is the union of members of $X / \rho,[2]$.

The equivalence relation $\rho$ is upper semicontinuous (almost upper semicontinuous) if for each $D \in X / \rho$ and any open (regular open) neighborhood $U$ of $D$ in $X$ there is an open admissible set $V$ such that

$$
D \subset V \subset U . \quad[2]
$$

\section{Results}

Definicija 2.1. An open cover $\mathcal{U}$ is $\delta$-even if there exists a $\tau_{p}^{*}$-open neighborhood $V$ of the diagonal in $\left(X \times X, \tau_{p}\right)$, (where $\tau_{p}$ is the product topology), such that $V[x] \subset U$ for some $U \in \mathcal{U}$.

Theorem 1. An almost-regular space $(X, \tau)$ is nearly paracompact iff every regular open cover is $\delta$-even.

Proof. Necessity: Let $\mathcal{U}=\left\{U_{\alpha}: \alpha \in I\right\}$ be any regular open covering of $X$.

Since $X$ is almost-regular and nearly paracompact, there exists by Theorem 1.6 in [15], a regular open star refinement $\mathcal{V}=\left\{V_{\beta}: \beta \in J\right\}$. Let $V=U\left\{V_{\beta} \times V_{\beta}: \beta \in J\right\}$. Then $V$ is $\tau_{\rho}^{*}$-open neighborhood of the diagonal in $X \times X$. We shall prove that for each $x \in X, V[x] \subset U_{\alpha}$ for some $\alpha \in I$.

If $y \in V[x]$, then $(x, y) \in V$ and hence there exists $\beta \in J$ such that $(x, y) \in V_{\beta} \times V_{\beta}$. Then $x \in V_{\beta}$ and $y \in V_{\beta}$. Hence, $y \in \operatorname{St}(x, \mathcal{V})$. Then, $V[x] \subset S t(x, \mathcal{V})$. Since $\mathcal{V}$ is star refinement of $\mathcal{U}$, therefore there exists $\alpha \in I$ such that $V[x] \subset S t(x, \mathcal{V}) \subset U_{\alpha}$. Hence the result. 
Sufficiency: We shall prove that $\left(X, \tau^{*}\right)$ is paracompact.

Let $\mathcal{G}=\left\{G_{\alpha}: \alpha \in I\right\}$ be any $\tau^{*}$-open covering of $X$. Then, there exists a $\tau$-regular open refinement $\mathcal{U}=\left\{U_{\beta}: \beta \in J\right\}$ of $\mathcal{G}$.

Since, every regular open cover is $\delta$-even, therefore there exists a $\tau_{p}^{*}$ open neighborhood $V$ of diagonal in $X \times X$ such that for each $x \in X, V[x] \subset U_{\beta}$ for some $\beta \in J$. Then, $\left(X, \tau^{*}\right)$ is the space, which is regular and every $\tau^{*}$-open cover is even, therefore $\left(X, \tau^{*}\right)$ is paracompact by Theorem 5.28 in [2].

Hence $(X, \tau)$ is nearly paracompact.

It would be interesting to see that $\rho$ is closed in $X^{2}$, where $\rho$ is an equivalence relation in $X$. Some results will be given in that sense.

In [2] the following result is proved.

Theorem A. If $X / \rho$ is a Hausdorff space, then $\rho$ is closed in $X^{2}$. If the projection $P: X \cdot X / \rho$ is an open mapping and $\rho$ is a closed subset of $X^{2}$, then $X / \rho$ is Hausdorff.

This result will be used in the proves of some theorems.

Theorem 2. Let $(X, \rho)$ be a graph, where $\rho$ is an upper semicontinuous equivalence relation in $X$. For each $x \in X$, let the equivalence class $\rho(x)(X \in$ $\rho(x))$ be an $\alpha$-Hausdorff $\alpha$-paracompact subset of $X$. Then $\rho$ is closed in $X^{2}$.

Proof. It follows that the quotient space $X / \rho$ is Hausdorff (Theorem 2.1 in [11]. Thus, by Theorem A, $\rho$ is closed in $X^{2}$.

Theorem 3. Let $X$ be any space. Let $A$ be a compact subset of $X$ such that every two points of $A$ can be strongly separated by open subsets of $X$. Let $X \backslash A$ be an $\alpha$-Hausdorff $\alpha$-nearly paracompact subset of $X$. Let $\rho$ be an equivalence relation in $X$ defined as follows

$$
\rho=\{(x, y): x, y \in X \backslash A \quad \text { or } \quad x=y\} .
$$

Then the quotient space $X / \rho$ is Hausdorff and compact. $\rho$ is closed in $X^{2}$.

Proof. The equivalence relation $\rho$ is upper semicontinuous such that $\rho(x)$ is $\alpha$-Hausdorff $\alpha$-nearly paracompact, for each $x \in X$. Thus, $X / \rho$ is Hausdorff. Hence $\rho$ is closed in $X^{2}$.

Now, let

$$
\mathcal{U}=\left\{U_{i}: i \in I\right\}
$$

be any open covering of $X / \rho$. Then

$$
\left\{P^{-1}\left(U_{i}\right): i \in I\right\}
$$

is an open covering of $X$. Since $A$ is compact, there is a finite subset $I_{0}$ of $I$ such that

$$
A \subset \cup\left\{P^{-1}\left(U_{i}\right): i \in I_{0}\right\}
$$


Let $U^{*}$ be such element of $\mathcal{U}$ such that

$$
P(X \backslash A) \subset U^{*} .
$$

$(X \backslash A$ is a point of the quotient space $X / \rho)$. Since

$$
X=\cup\left\{P^{-1}\left(U_{i}\right): i \in I_{0}\right\} \cup P^{-1}\left(U^{*}\right)
$$

it follows that the family

$$
\left\{U_{i}: i \in I_{0}, U^{*}\right\}
$$

is a finite open covering of $X / \rho$, hence $X / \rho$ is compact.

Theorem 4. Let $X$ be any regular space. Let $X$ be an open subset of $X$ such that every two points of $A$ can be strongly separated by open subsets of $X$. Let $X \backslash A$ be $\alpha$-Hausdorff. Let $\rho$ be an equivalence relation in $X$ defined by

$$
\rho=\{(x, y): x, y \in X \backslash A \text { or } x=y\} .
$$

Then $\rho$ is closed in $X^{2}$.

Proof. It would be proved that $X / \rho$ is $a$-Hausdorff space. If $m$ and $n$ are two points of $A$, then there are open sets $U$ and $V$ of $X$ such that

$$
m \in U, n \in V, U \cap V=\emptyset .
$$

Let

$$
U_{1}=U \cap A, V_{1}=V \cap A .
$$

Then $P\left(U_{1}\right)$ and $P\left(V_{1}\right)$ are disjoint open sets of $X / \rho$ containing $P(m)$ and $P(n)$ respectively.

Now, let

$$
m \in A \text {. }
$$

Since $X \backslash A$ is $\alpha$-regular, it follows that there are two open subsets $U$ and $V$ of $X$ such that

$$
x \in U, X \backslash V, U \cap V=\emptyset .
$$

Then

$$
P(U) \text { and } P(V)
$$

are two disjoint open subsets of $X / \rho$ containing $P(m)$ and $P(X \backslash A)$ respectively. Hence $X / \rho$ is Hausdorff. By Theorem A it follows that $\rho$ is closed in $X^{2}$.

In [2] the following result is proved. 
Theorem B. If $f_{1}$ and $f_{2}$ are two continuous mappings of a space $X$ into a Hausdorff space $Y$, then

$$
F=\left\{x: f_{1}(x)=f_{2}(x)\right\}
$$

is closed in $X$. If $F$ is a dense subset of $X(C 1 F=X)$ then $f_{1}=f_{2}$.

Similar results is not always true for two equivalence relation in $X$, as it is shown by the following example

Example 1. Let $R$ be the real line (the set of all real numbers endowed with the usual topology in $P$ ). Define an equivalence relation $\rho_{1}$ in $R$ as

$$
\rho_{1}=\{(x, y): x, y \in[0,1] \text { or } x=y\} .
$$

Define an equivalence relation $\rho_{2}$ in $R$ as follows

$$
\rho_{2}=\{(x, y): x, y \in[0,2] \text { or } x=y\} .
$$

The equivalence relation $\rho_{1}$ is upper semicontinuous such that, for each $x \in R, \rho_{1}(x)$ is an $\alpha$-Hausdorff compact subset of $R$, thus $R / \rho_{1}$ is Hausdorff. Hence $\rho_{1}$ is closed in $R^{2}$.

The equivalence relation $\rho_{2}$ is upper semicontinuous such that, for each $x \in R, \rho_{2}(x)$ is an $\alpha$-Hausdorff compact subset of $R$, thus $R / \rho_{2}$ is Hausdorff. Hence $\rho_{2}$ is closed in $R^{2}$.

Now, we have

$$
\rho_{1}(x)=\rho_{2}(x) \text { for each } x \in A=R \backslash[0,2]
$$

but the subset $A$ is not closed in $R$.

Theorem 5. Let $\rho_{1}$ and $\rho_{2}$ be any two equivalence relation in $X$ and let

$$
\mathcal{U}=\left\{\rho_{1}(x): \rho_{1}(x)=\rho_{2}(x)\right\} .
$$

If each member of $\mathcal{U}$ is an $\alpha$-Hausdorff $\alpha$-paracompact subset and $\mathcal{U}$ is locally finite, then

$$
U=\cup\left(\rho_{1}(x): \rho_{1}(x) \in \mathcal{U}\right\}
$$

is closed in $X$. If $U$ is dense, then $\rho_{1}=\rho_{2}$, i.e. $X / \rho_{1}=X / \rho_{2}$.

Proof. Since every $\alpha$-Hausdorff $\alpha$-paracompact subset is closed ([11]) it follows that $\rho_{1}(x)$ is closed for each $\rho_{1}(x) \in \mathcal{U}$. Since $\mathcal{U}$ is locally finite, it follows that

$$
\begin{aligned}
& C 1 U=C 1 \cup\left\{\rho_{1}(x): \rho_{1}(x) \in \mathcal{U}\right\}=\cup\left\{C 1 \rho_{1}(x): \rho_{1}(x) \in \mathcal{U}\right\}= \\
& =\cup\left\{\rho_{1}(x): \rho_{1}(x) \in \mathcal{U}\right\}=U .
\end{aligned}
$$

Since, any union of $\alpha$-Hausdorff subsets is $\alpha$-Hausdorff, thus $U$ is $\alpha$-Hausdorff. If $U$ is dense in $X$, then $C 1 U=X$. Thus

$$
\rho_{1}=\rho_{2} \text { i.e. } X / \rho_{1}=X / \rho_{2} \text {. }
$$




\section{Some examples of quotient spaces}

Example 2. Let $\equiv_{1}$ be a congruence in the space $R$

$$
\left(x \equiv_{1} y\right)=((\exists n \in Z) x=y+n) .
$$

Every equivalence class is a closed paracompact subset of $R$. The projection $P: R \rightarrow R / \equiv_{1}$ is an open (not a closed) mapping. The space $R / \equiv_{1}$ is Hausdorff. Thus, $\equiv_{1}$ is closed in $R^{2}$.

Example 3. Let

$$
A=[0,1] \subset R
$$

have a subspace topology with respect usual topology. Let $\equiv_{1}$ be a congruence in the space $R$.

Every equivalence class is a closed compact subset of $A$. The projection $P: A \rightarrow A / \equiv_{1}$ is a closed (not an open) mapping. The space $A / \equiv_{1}$ is Hausdorff, hence $\equiv_{1}$ is closed in $A^{2}$ i.e. in $R^{2}\left(A^{2}\right.$ is closed in $\left.R^{2}\right)$. Since every continuous image of compact space is compact, it follows that $A / \equiv_{1}$ is compact.

Example 4. Let

$$
A=[-1,1] \subset R
$$

have a subspace topology with respect usual topology. Define an equivalence relation $\rho$ in $A$ by taking

$$
\rho=\{(x, y): y=x \vee y=-x \text { for } x \in(-1,1)\} \cup\{(1,1),(-1,1)\} .
$$

Every equivalence class is a closed compact subset of $A$. The projection $P: A \rightarrow A / \rho$ is an open mapping, hence $A / \rho$ is not Hausdorff ( $\rho$ is not closed in $\left.A^{2}\right) . A / \rho$ is $T_{1}$ (every point is closed).

Example 5. Define an equivalence relation $\rho$ in $R$ as follows

$$
\rho=\{(x, y): x, y \in Z \text { or } x=y\} .
$$

Every equivalence class is a closed $\alpha$-paracompact subset of $R$.

It follows that the quotient space $R / \rho$ is Hausdorff, thus $\rho$ is closed in $R^{2}$. The space $R / \rho$ is not locally compact (for point $P(Z)$ there is not an open subset $V$ such that $C 1 V$ is compact in $R / \rho$ ).

Example 6. Let

$$
A=[0, \infty] \subset R
$$

have a subspace topology with respect usual topology. Define an equivalence relation $\rho$ by taking

$$
\rho=\{(x, y): y=x \vee y=1 / x \text { for } x \notin 0\} \cup\{(0,0)\} .
$$

Every equivalence class is a closed compact subset of $A$. $\rho$ is closed in $A^{2}$. The projection $P$ is an open (not a closed) mapping). Hence $A / \rho$ is Hausdorff. The space $A / \rho$ is compact. 


\section{References}

[1] T.R. Hamlet and L.L. Herrington, The closed graph and P-closed graph properties in general topology, Amer. Math. Soc. Providence R.I. (1981), 1-68.

[2] J.L. Kelley, General Topology, Van Nostrand, Princeton, N.J., 1961.

[3] I. Kovačević, A note on almost closed mappings and nearly paracompactness, Univ. u N. Sadu, Zb. rad. Prir. mat. Fak. Mat. Ser. 13 (1983), 87-101.

[4] I. Kovačević, A note on quotient spaces and paracompactness, Univ. u N. Sadu, Zb. rad. Prir. mat. Fak. Mat. Ser. 11 (1981), 89-99.

[5] I. Kovačević, On $\alpha$-Hausdorff subsets, almost closed mappings and almost upper semicontinuous decomposition, Indian J. Pure Appl. Math. 20(4) (1989), 334340.

[6] I. Kovačević, On almost closed mappings, paracompactness and partial equivalence relations, Indian J. Pure Appl. Math. 25(9) (1994), 949-954.

[7] I. Kovačević, On $M_{N}$ subsets, J. Math. Novi Sad 27(1) (1997), 45-52.

[8] I. Kovačević, On subsets, almost closed mappings and paracompactness, Glasnik matematički 24(44) (1989), 125-132.

[9] I. Kovačević, Some properties of $M_{N}$ subset and almost closed mappings, Indian J. Pure Appl. Math. 27(9) (1996), 875-881.

[10] I. Kovačević, Some properties of nearly and almost compact spaces, Math. Balkanica 7 (1977), 195-200.

[11] I. Kovačević, Subsets and paracompactness, Zb. rad. Prir. mat. Fak. Univ. u N. Sadu 14(2) (1984), 79-87.

[12] T. Noiri, Almost-continuity and some separation axioms, Glasnik matematički 9(29) (1974), 131-135.

[13] M.K. Singal and A.R. Singal, Almost continuous mappings, Yokohoma Math. J. 16(1968), 63-73.

[14] M.K. Singal and Asha Mathur, On nearly compact spacesII, Boll. Un. Mat. Ital. (4) 2(1969), 702-710.

[15] M.K. Singal and S.P. Arya, On nearly paracompact spaces, Matem. vesnik 6(21) (1969), 3-16.

Faculty of Engineering University of Novi Sad

Trg D. Obradovića 6 21000 Novi Sad Serbia \& Montenegro 